\title{
Comparative Study of Efficacy and Safety of Coded Unani Formulations-UNIM-00 I + UNIM-003 with Methoxsalen in Cases of Bars (Vitiligo)
}

\author{
Parvez Khan', Radhey Shyam Verma ${ }^{2}$, Sadia Ayub ${ }^{3}$, Sheereen Afza ${ }^{4}$, Jamal Akhtar ${ }^{5}$,
} Asim Ali Khan ${ }^{6}$

\begin{abstract}
${ }^{1,2,3,4}$ Regional Research, Institute of Unani Medicine (CCRUM), Shahjahan Manzil, Near Riding Club, Qila Road Aligarh, U.P., India. ${ }^{5,6}$ Central Council for Research in Unani Medicine, Jawaharlal Nehru Anusandhan Bhavan, Institutional Area, Opp. D-Block Janakpuri, New Delhi, India.
\end{abstract}

DOI: https://doi.org/10.24321/2394.6547.202009

I $\quad \mathbf{N} \quad \mathbf{F} \mathbf{O}$

Corresponding Author:

Parvez Khan, Institute of Unani Medicine (CCRUM), Shahjahan Manzil, Near Riding Club, Qila Road Aligarh, U.P., India.

E-mail Id:

waytoparvez@gmail.com

Orcid Id:

https://orcid.org/0000-0001-6380-002X

How to cite this article:

Khan P, Verma RS, Ayub S, Afza S, Akhtar J, Khan AA. Comparative Study of Efficacy and Safety of Coded Unani Formulations-UNIM001+UNIM-003 with Methoxsalen in Cases of Bars (Vitiligo). J Adv Res Ayur Yoga Unani Sidd Homeo 2020; 7(3\&4): 16-26.

Date of Submission: 2020-12-08

Date of Acceptance: 2020-12-27

\section{$\begin{array}{llllllll}\mathbf{A} & \mathbf{B} & \mathbf{S} & \mathbf{T} & \mathbf{R} & \mathbf{A} & \mathbf{C} & \mathbf{T}\end{array}$}

Background: Vitiligo is a common acquired disorder of skin depigmentation in varying patterns, varying from small maculae's with scalloping borders to near total depigmentation of body. The prevalence of vitiligo in India has been invariably reported between $0.25 \%$ and $4 \%$ of dermatology outpatients across studies from India and up to $8.8 \%$ in Gujarat and Rajasthan. The study was conducted to compare the coded Unani formulation UNIM-001 (tablet) and UNIM-003 (ointment) with Melanocyl tablet (10mg each) (Methoxsalen) as standard control.

Methodology: Sixty Five patients of trial group (UNIM-001+ UNIM003) and sixty seven patients of control group (Comparator group) of 12-50 years of either sex were selected from patients attending the Out Patient Department (OPD), at RRIUM, Aligarh during 2015-18. The patients were treated for eight months with instructions and are followed every month for efficacy and safety of the drug(s). The CRF was maintained, and post treatmet followup was done at 3 months for the repigmentaion retained by the patient.

Result: The UNIM-001 (tablets) and UNIM -003 (ointment) and standard control drug Melanocyl tablets (methoxsalen) showed same results and did not show any side effects.

Conclusion: The trial drug and the standard control drugs both possess same anti- vitiligo efficacy and are also safe for the patients.

Keywords: Vitiligo (Bars), Temperament (Mizaj), Dermatomal, Non-dermatomal Bilateral symmetrical

\section{Introduction}

Vitiligo is a common acquired disorder of skin depigmentation in varying patterns, varying from small maculae's with scalloping borders to near total depigmentation of body. The disorder affects nearly $1 \%-2 \%$ of the world population 
irrespective of race and ethnicity with highest incidence recorded in Indian subcontinent followed by Mexico and Japan. ${ }^{1,2,4}$ Vitiligo occurs worldwide with an overall prevalence of $1 \%$. However, its incidence varies from 0.1 to > $8.8 \% .^{3}$ The prevalence of vitiligo in India has been invariably reported between $0.25 \%$ and $4 \%$ of dermatology outpatients across studies from India and up to $8.8 \%$ in Gujarat and Rajasthan. ${ }^{2,5,6}$ It has a familial incidence of 25 to $30 \% .^{7} \mathrm{~A}$ multitude of plausible theories have been put forward to explain the pathogenesis of vitiligo and mechanisms that finally lead to the loss of functional melanocytes from the epidermis. ${ }^{8}$ The important ones include a genetic predisposition, autoimmune destruction of melanocytes, ${ }^{9}$ zinc- $\alpha 2$-glycoprotein deficiency hypothesis, ${ }^{10}$ altered redox status $^{11}$ and free radical mediated melanocytes damage, ${ }^{12}$ heightened sympathetic response and catecholamine's/ neurotransmitter mediated melanocytes damage ${ }^{13}$ and impaired melanocytes adhesion or melanocytorrhagy. ${ }^{14}$ The fusion of all these effectively explains the vitiligo pathogenesis the combination theory. ${ }^{15}$ The medication of vitiligo comprises medical, phototherapeutic and surgical modalities. In some situations, a combination of these methods works the best. ${ }^{16}$ The clinically characteristic symptoms of the vitiligo are pale or milk-white maculae or patches due to the selective destruction of melanocytes. They occur on the skin in different parts of the body and sometimes also on the mucous membranes. ${ }^{17}$

In modern medicine system various therapy such as topical corticosteroids, ${ }^{18-21}$ systemic drug: Methotrexate vs. maniple dexamethasone and minocycline vs. minipulse dexamethasone, ${ }^{22,23}$ ultraviolet light therapy, ${ }^{24}$ Monochromatic Excimer Light laser (MEL) therapy, ${ }^{25}$ combination of UV and topical or systemic therapies ${ }^{26}$ and combination of Monochromatic Excimer Light laser (MEL) and topical therapies ${ }^{27}$ have recently been used for treatment of vitiligo, but they all have certain side effects.

Most of the Unani physicians including Jālinūs, as mentioned in Mu,ōlajāt-i Buqrāțiyāh, ${ }^{28}$ Ibn Sinā in Al-Qānūn fi"l-Ṭib, ${ }^{29}$ Jurjānīin his book Dhakhīra Khawārizm Shāhī, ${ }^{30}$ Hakīm Akbar Arzānī in his book Țibb-i Akbar, ${ }^{31}$ and Sadīd al-Dīn Gāzrūni in the book Al-Sadīdī, ${ }^{32}$ described the cause of vitiligo as Du,af-i Quwwat-i Mughayyira-i Badan (transformative faculty, ${ }^{33}$ the power that brings changes, and shapes the nutrients into tissues) and Mushabbiha-i Badan (power of resemblance ${ }^{34}$ ). This $D u$, af (weakness) may be due to accumulation of Balgham-i Ghalīz (viscous phlegm), Fasād al-Dam or Barūdat al-Dam in the body.

In Unani System of Medicine (USM), many single drugs such as Habbul-Neel (Ipomoea nil), Atrilal (Ammi majus), Babchi (Psoralea corylifolia), Panwar (Cassia tora), Kharbaq Syah (Helleborus niger), Sheetraj (Plumbago zeylanica), Saqmooniya (Convulvulus scammonia) ${ }^{35-37}$ as well as compound formulations (systemic) Safoof Bars ${ }^{38}$ topical application: Zamad Bars have been used in the therapeutic management of vitiligo.

\section{Methodology}

The study was conducted between 2015 and 2018 at RRIUM, Aligarh. The coded Unani formulation UNIM-001+ UNIM003 was procured from Central Council for Research in Unani Medicine, New Delhi. The UNIM-001 is in tablet forms and UNIM-003 is in ointment form. Melanocyl tablet (10mg each) (Methoxsalen) was purchased from Aligarh. Sixty Five patients of trail group (UNIM-001+ UNIM-003) and sixty seven patients of control group (Comparator group) of 12-50 years of either sex were selected from patients attending the Out Patient Department (OPD). Study participant will be asked not to take any anti-vitiligo therapy during the trial period. The study is a single blind study. Participants are allocated to the treatment group by pre-randomized schedule. One group was received (UNIM001+ UNIM-003) and other group was received Melanocyl Tablet (10mg each) (Methoxsalen) as a comparator drug.

Total 260 cases were screened and 260 cases were registered on the basis of inclusion and exclusion criteria, 65 and 67 cases completed the study in both the group (trail and control group) respectively. The efficacy and safety of Unani formulation (UNIM-001+ UNIM-003) as a trial drug and allopathic drug Melanocyl Tablet (Methoxsalen) as a comparator drug were evaluated on the basis of biochemical, haematological parameters as well as percentage repigmentation response.

\section{Selection Criteria}

Patients of were enrolled on the basis of following inclusion and exclusion criteria:

\section{Inclusion Criteria}

- Patients with clinically diagnosed segmental and nondermatomal vitiligo.

- Patients of either sex in the age group 12-50 years.

- Patients with any duration of disease, site, extension and distribution of lesions.

- Willingness to give written informed consent form.

\section{Exclusion Criteria}

- Patients with active Vitiligo treatment with other drugs/ systems.

- Non-cooperative patients.

- History of drug or alcohol abuse, chronic smokers not willing to abstain from smoking during the study period.

- Any clinically significant abnormality identified on physical examination or laboratory Tests.

- Patients having any systemic disease and other skin diseases.

- $\quad$ Patients with known allergies. 
- Patients with impaired cardiac, hepatic and renal function.

- Patients with history of malignancy.

- Concomitant use of any other antioxidants.

- Patients with history of hypersensitivity to any other investigational drugs/ herbal medicine.

- Patients receiving any other investigational product within 4 weeks.

- $\quad$ Patients with any medical condition, where physician feels participation in the study could be detrimental to patients well being.

- Patients with uncontrolled infection

- Pregnant and lactating women.

\section{Ethical Consideration}

All patients were included in the study after obtaining written informed consent and study was conducted. This research study is also registered under Clinical Trial ResistryIndia (CTRI) (CTRI/ 2013/ 12/ 004215 dated 13/ 12/ 2013.

\section{Drug, Dose and Mode of Administration}

UNIM-001 (800 mg each) was given to the patients in a dose of one to four tablets orally two-three times a day after meal for a period of 240-days. UNIM-003 topical drug were in lotion form given for topical application and it was applied every alternate day followed by exposure to sun light. Patients were advised to apply on one patch and expose to sunlight at least for 3-5 days in order to ascertain the sensitivity of an individual, based on further application may be planned. The exposure time was adjusted according to the skin sensitivity of an individual permits which could range from 3-30 minutes in the early hours in the morning.

Melanocyl Tablet (10mg each) (Methoxsalen) was given orally two tablet BD after meal.

Treatment allocation: RRIUM had 20 patients per block. Block randomization technique was used to allocate the treatment schedule to the patients. Pre-randomization was done by using statistical graph pad. Randomization chart will be kept with a third person who is not involved directly in the trial.

Blinding: The assessor will not know the treatment allocation the patients.

\section{Assessment of Mizaj (Temperament)}

Assessment of Mizāj (Temperament) was done at baseline.

\section{Follow-up evaluation}

The patients will be assessed clinically at day $0,30^{\text {th }}$-day, $60^{\text {th }}$-day, $90^{\text {th }}$-day, $120^{\text {th }}$ - day, $150^{\text {th }}$-day a $180^{\text {th }}$ - day, $210^{\text {th }}$ day and $240^{\text {th }}$-day. The subjective and objective clinical observations were recorded in the follow-up sheet.

\section{Criteria for Assessment of Efficacy}

To assess the response of treatment in patients of Vitiligo, the following parameters were used.

1. Efficacy evaluation: By observation and percentage Repigmentation by digital photography.

2. Safety Evaluation:

- Adverse drug reaction/ Adverse drug event was reported by the patients in the case report form. It was reported to the Institutional Ethics committee (IEC) 39. IEC was reviewed according to its SOP.

- Biochemical and hematological investigation were carried out at baseline and once a month at every follow-up.

\section{Assessment of safety}

\section{Biochemical Analysis}

Serum Glutamate Pyruvate Transaminase (SGPT, E.C. 2.6.1.2) and Serum Glutamate Oxaloacetate Transaminase (SGOT, E.C. 2.6.1.1.) were done by the method described by International Federation of Clinical Chemistry (IFCC) 40 , Blood Urea by the method of Tiffany et al. ${ }^{41}$ Serum Creatinine by Bowers method, ${ }^{42}$ Serum Total Bilirubin by Pearlman and Lee, ${ }^{43}$ Total protein by Biuret method and point, ${ }^{44}$ Albumin by BCG Dye method and point. ${ }^{45}$

\section{Haematological Analysis}

Haematological parameters were done according to the method described by Mukherjee. ${ }^{45}$ It included Haemoglobin (Hb), Erythrocyte Sedimentation Rate (ESR), Total Leucocytes Counts (TLC), Red Blood Corpuscles (RBC), Platelets Count and Differential Leucocytes Counts (DLC): Polymorphs, Lymphocyte and Eosinophil Counts.

\section{Collection of Blood Serum}

Blood samples were collected by puncturing the vein at each investigation. $1.0 \mathrm{ml}$ of blood with Ethylene Diamine Tetra Acetic Acid (EDTA) was used for various haematological parameters and another 2.0-2.5 $\mathrm{ml}$ of blood sample was allowed to clot and serum was separated by centrifugation, which was used for various biochemical parameters. Biochemical and haematological investigations were carried out.

\section{Statistical Analysis}

Data were analyzed statistically by one-way Analysis of Variance (ANOVA) followed by Dennett's' test. The values were considered significant when the P-value was found less than 0.05 .

\section{Result and Discussion}

\section{Clinical Findings}

\section{Demographic Study}

Out of 65 patients of Vitiligo (Bars) of trail group, 32 (49.23\%), mean age 27.06 years were male and 33 (50.77\%), 
mean age 27.06 years were female and out 67 patients of Vitiligo (Bars) of control group 26 (38.81\%), mean age 27.92 years were male and 41 (61.19\%), mean age 20.90 years were female which shows that females of age group (12-20 years) have higher incidence 17 (26.15\%) with mean age 16.18 years and $27(40.30 \%)$ with mean age 16.44 years as compared to male in both the groups (Trail and control group) respectively. Shah et.al., $2008^{46}$ had reported similar type of observations.

21-40 years of trial group 16 (24.62\%) with mean age 30.25 years were male and 15 (22.39\%) with mean age 31.12 years were female, whereas out 67 patients of Vitiligo (Bars) of control group 15 (22.39\%) with mean age 31.12 years were male and 13 (19.40\%) with mean age 28.42 years were female.

41-50 years of trial group 04 (6.15\%) with mean age 45.0 years were male and $04(6.15 \%)$ with mean age 47.5 years were female, whereas out 67 patients of Vitiligo (Bars) of control group 03 (4.48\%) with mean age 46.33 years were male and 01 (1.49\%) with mean age 50.00 years were female (Table 1).

The age of onset of Vitiligo ranged from 12 to 20 years of female of trail group and control group have higher incidence 22 (33.85\%) (Mean age 10 years) and 35 (52.24\%) (Mean age 12 years) respectively, whereas the age of onset of Vitiligo of male of both trail and control group have similar incidence. The age of onset of Vitiligo ranged from 21 to 40 years of male of trail group and control group have higher incidence 15 (23.08\%) (Mean age 29 years) and 11 (16.42\%) (Mean age 32 years) respectively. Trail group of 17 (26.15\%) patients, mean age 25.18 year had family history and $48(73.85 \%)$ patients, mean age 25.94 years had no history, whereas in Control group of 17 (25.37\%), patients, mean age 21.65 years had family history and 50 (74.63\%) patients, mean age 24.3 years had no history. Sehgal and Srivastava, $2007^{47}$ had observed similar type of results.

In socio-economic status study, Out of 65 patients of Vitiligo (Bars) of trail group, 47 (72.31\%) patients, mean age 24.64 years were lower income group followed by middle income group 17 (26.15\%) patients, mean age 29.35 years and higher income group 01 (1.54\%) patients, 16.00 years. In total 67 patients of control group 50 (74.63\%) patients, mean age 23.54 years were lower income group followed by middle income group 17 (25.37\%) patients, mean age 24.59 years. Lower income group in both groups have higher incidence than middle income group. Similar type of observation had been reported by Almomani et. al., 2015.48

Non-vegetarian patients of Vitiligo of trial group as well as control group had more incidences 39 (60\%) and 43 (66.15\%) than vegetarian $26(40.00 \%)$ and 24 (35.86\%) respectively (Table 2 ) Jimi et al., $2011^{49}$ had reported similar type of observation.
Both trial as well as control group patients had a history of previous treatment in different system of medicine. Out of 65 patients of Vitiligo (Bars) of trail group, 05 (7.69\%) patients, mean age 21.6 years had tried Allopathic treatment, 23 (35.39\%) patients, mean age 26.61 years were received Unani treatment, $07(10.77 \%)$ patients, mean age 26.14 years were received Ayurvedic treatment and 30 $(46.15 \%)$ patients, mean age 25.67 had without treatment.

Out of 65 patients of Vitiligo (Bars) of control group, 06 (8.96\%) patients, mean age 23.67 years had tried Allopathic treatment, $32(47.76 \%)$ patients, mean age 22.50 years were received Unani treatment, 05 (7.46\%) patients, mean age 25.40 years were received Ayurvedic treatment and 24 (35.82\%) patients, mean age 24.75 had without treatment (Table 1).

\section{Temperament (humors) Study}

In both trial and control group the incidence of Balghami (Phlegmatic) temperament (humors) affected with Vitiligo were 65 (100.00\%), mean age 25.74 years and 66 (98.51\%), mean age 23.30 years respectively. Balghami (Phlegmatic) temperament had higher incidence. Thulasamma et al., $2017^{50}$ had reported similar type of observation (Table 1 ).

\section{Clinical Patterned}

Out of 65 patients of Vitiligo (Bars) of trail group, 29 (44.62\%), mean age 26.10 years were dermatomal type of Vitiligo and 36 (55.39\%), mean age 25.44 years were non-dermatomal type of Vitiligo whereas in total 67 patients of control group 34 (50.75\%), mean age 23.56 years were dermatomal type of Vitiligo and 33 (49.25\%), mean age 23.70 years were non-dermatomal type of Vitiligo (Table 1). Non-dermatomal type of Vitiligo had more incidences in trail group. Similar results had been reported by an authors. ${ }^{51}$ In control group dermatomal type of Vitiligo had more incidences. Similar inference had been found by Khaitan et al. ${ }^{52}$

Out of 65 patients of Vitiligo (Bars) of trail group, 37 (56.92\%) patients, mean age 26.62 years had extensive pattern of lesion and 28 (43.08\%) patients, mean age 24.17 years had non-extensive type of lesion. In total 67 patients of control group 35 (52.24\%) patients, mean age 24.57 years had extensive type of lesion and 32 (47.76\%) patients mean age 23.70 years had non-extensive type of lesion. In both the group, extensive pattern of lesions had more incidences than non extensive type.

Out of 65 patients of Vitiligo (Bars) of trail group, 23 (35.39\%) patients, mean age 25.96 years had unilateral type of distribution of lesion and 25 (38.46 \%) patients, mean age 25.20 years had bilateral type of distribution of lesion, 16 (24.62\%) patients, mean age 26.00 years had bilateral symmetrical type of distribution of lesion and 01 (1.54\%) patients, mean age 30.00 years had had bilateral 
asymmetrical type of distribution of lesion whereas in total 67 patients of control group 24 (35.82\%) patients, mean age 24.62 years had unilateral type of distribution of lesion, 21 (31.34\%) patients, mean age 23.43 years had bilateral type of distribution of lesion, 22 (32.84\%) patients, mean age 23.83 years had bilateral symmetrical type of distribution of lesion (Table 1).

Table I.Demographic study of Vitiligo

\begin{tabular}{|c|c|c|c|c|}
\hline & $\begin{array}{c}\text { Trial Group } n=65, \\
\% \text { age }\end{array}$ & Mean age $\pm S . D$. & $\begin{array}{l}\text { Control Group } \\
\text { n=67, \%age }\end{array}$ & Mean age \pm S.D. \\
\hline \multicolumn{5}{|c|}{ Sex Wise } \\
\hline Female & $33(50.77 \%)$ & $25.21 \pm 10.66$ & $41(61.19 \%)$ & $20.90 \pm 8.14$ \\
\hline Male & $32(49.23 \%)$ & $27.06 \pm 12.32$ & $26(38.81 \%)$ & $27.92 \pm 11.06$ \\
\hline \multicolumn{5}{|c|}{ Age wise distribution (in years) } \\
\hline $12-20$ & $\begin{array}{c}\text { F } 17(26.15 \%) \\
\text { M } 12(18.46 \%)\end{array}$ & $\begin{array}{l}16.18 \pm 2.40 \\
15.75 \pm 2.34\end{array}$ & $\begin{array}{l}\text { F } 27(40.30 \%) \\
\text { M } 08(11.94 \%)\end{array}$ & $\begin{array}{l}16.44 \pm 2.49 \\
14.88 \pm 2.36\end{array}$ \\
\hline $21-40$ & $\begin{array}{l}\text { F } 12(18.46 \%) \\
\text { M } 16(24.62 \%)\end{array}$ & $\begin{array}{l}30.77 \pm 6.44 \\
30.25 \pm 5.79 \\
\end{array}$ & $\begin{array}{l}\text { F } 13(19.40 \%) \\
\text { M } 15(22.39 \%)\end{array}$ & $\begin{array}{l}28.42 \pm 6.22 \\
31.12 \pm 7.18\end{array}$ \\
\hline $41-50$ & $\begin{array}{l}\text { F } 04(6.15 \%) \\
\text { M } 04(6.15 \%)\end{array}$ & $\begin{array}{c}45.00 \pm 2.94 \\
47.50 \pm 3.5\end{array}$ & $\begin{array}{l}\text { F } 01(1.49 \%) \\
\text { M } 03(4.48 \%)\end{array}$ & $\begin{array}{c}50.00 \\
46.33 \pm 3.21\end{array}$ \\
\hline \multicolumn{5}{|c|}{ Age onset of the disease } \\
\hline $12-20$ & $\begin{array}{l}\text { F } 22(33.85 \%) \\
\text { M } 17(26.15 \%)\end{array}$ & $\begin{array}{l}10.14 \pm 4.08 \\
14.06 \pm 3.96\end{array}$ & $\begin{array}{l}\text { F } 35(52.24 \%) \\
\text { M } 15(23.40 \%)\end{array}$ & $\begin{array}{l}12.29 \pm 4.39 \\
12.33 \pm 5.23\end{array}$ \\
\hline $21-40$ & $\begin{array}{l}\text { F } 06(9.23 \%) \\
\text { M } 15(23.08 \%)\end{array}$ & $\begin{array}{l}31.00 \pm 6.95 \\
28.92 \pm 4.98\end{array}$ & $\begin{array}{c}\text { F } 05 \text { (7.50\%) } \\
\text { M } 11(16.42 \%)\end{array}$ & $\begin{array}{l}29.33 \pm 5.85 \\
32.00 \pm 5.46 \\
\end{array}$ \\
\hline $41-50$ & $\begin{array}{l}\text { F } 03(4.62 \%) \\
\text { M } 02(3.08 \%)\end{array}$ & $\begin{array}{l}48.00 \pm 3.46 \\
43.50 \pm 2.12\end{array}$ & $\begin{array}{c}\text { F Nil } \\
\text { M } 01(1.49 \%)\end{array}$ & $\begin{array}{c}\text { Nil } \\
44.00 \pm 0.00\end{array}$ \\
\hline
\end{tabular}

\begin{tabular}{|c|c|c|}
\hline Variables & Trial Group (n=65, \%age) & Control Group ( $n=67, \%$ age) \\
\hline \multicolumn{3}{|l|}{ Family History } \\
\hline Yes & $17(26.15 \%)$ & $17(25.37 \%)$ \\
\hline No & $48(73.85)$ & $50(74.63 \%)$ \\
\hline \multicolumn{3}{|l|}{ Economic Status } \\
\hline High Economy group (HEG) & $01(1.54 \%)$ & Nil \\
\hline Medium Economy Group (MEG) & $17(26.15 \%)$ & $17(25.37 \%)$ \\
\hline Low Income Group (LEG) & $47(72.31 \%)$ & $50(74.63 \%)$ \\
\hline \multicolumn{3}{|l|}{ Dietary Habits } \\
\hline Non-Vegetarian & $39(60.00 \%)$ & $43(66.15 \%)$ \\
\hline Vegetarian & $26(40.00 \%)$ & $24(35.82 \%)$ \\
\hline \multicolumn{3}{|l|}{ Types of Medication } \\
\hline Allopathic & $05(7.69 \%)$ & $06(8.96 \%)$ \\
\hline Unani & $23(35.39 \%)$ & $32(47.76 \%)$ \\
\hline Ayurvedic & $07(10.77 \%)$ & $05(7.46 \%)$ \\
\hline No medicine & $30(46.15 \%)$ & $24(35.82 \%)$ \\
\hline \multicolumn{3}{|l|}{ Assessment of Mizaj (Temperament) } \\
\hline Balghami (Phlegmatic) & 65 (100.00\%) & $66(98.51 \%)$ \\
\hline Safrawi (Bilious) & Nil & $01(1.49 \%)$ \\
\hline
\end{tabular}




\begin{tabular}{|c|c|c|}
\hline \multicolumn{3}{|l|}{ Clinical pattern of Vitiligo } \\
\hline Variables & Trial Group (n=65, \%age) & Control Group ( $n=67, \%$ age) \\
\hline \multicolumn{3}{|l|}{ Types of Vitiligo } \\
\hline Dermatomal & $29(44.62 \%)$ & $34(50.75 \%)$ \\
\hline Non-Dermatomal & $36(55.39 \%)$ & $33(49.25 \%)$ \\
\hline \multicolumn{3}{|l|}{ Extension of lesion } \\
\hline Extensive & 37 (56.92\%) & 35 (52.24\%) \\
\hline Non-Extensive & $28(43.08 \%)$ & $32(47.76 \%)$ \\
\hline \multicolumn{3}{|l|}{ Distribution of lesion } \\
\hline Unilateral & $23(35.39 \%)$ & $24(35.82 \%)$ \\
\hline Bilateral & $25(38.46 \%)$ & $21(31.34 \%)$ \\
\hline Bilateral symmetrical (B.S) & $16(24.62 \%)$ & $22(32.84 \%)$ \\
\hline Bilateral asymmetrical (B.AS) & $01(1.54 \%)$ & Nil \\
\hline
\end{tabular}

\section{Biochemical Studies}

\section{Liver Function Tests and Kidney Function Tests}

UNIM-001 (800 mg each) was given to the patients in a dose of two to four tablets orally two times a day after meal for a period of 240-days according to age. UNIM-003 topical drug were in lotion form given for topical application and it was applied every alternate day followed by exposure to sun light. Patients were advised to apply on one patch and expose to sunlight at least for 3-5 days in order to ascertain the sensitivity of an individual, based on further application may be planned. Melanocyl Tablet (10mg each) (Methoxsalen) was given orally two tablet BD after meal.

In both trial and control group, no significant alterations in liver function tests, kidney function tests as well as protein, albumin, globulin and $\mathrm{A} / \mathrm{G}$ ratio had been observed. Therefore, it can be inferred that it did not induce any negative or unfavorable response. The safety of the drug is therefore conformed (Table $2 \& 3$ ). Similar observation had been reported by other authors. . $3,54,55$

Table 2.Effect of Unani coded drug UNIM-00I + UNIM-003 on the levels of SGPT, SGOT, Bilirubin, Blood Urea and Serum Creatinine, Protein, Albumin, Globulin and A/G ratio in vitiligo patients

\begin{tabular}{|c|c|c|c|c|c|c|c|c|c|}
\hline $\begin{array}{c}\text { Group } \\
\text { Parameter }\end{array}$ & $\begin{array}{c}\text { BL } \\
\text { 0-Day }\end{array}$ & $\begin{array}{l}1^{\text {st }} F-U p \\
30^{\text {th }} \text { day }\end{array}$ & $\begin{array}{l}2^{\text {nd }} \text { F-up } \\
60^{\text {th }} \text { day }\end{array}$ & $\begin{array}{l}3^{\text {rd }} \text { F-up } \\
90^{\text {th }} \text { day }\end{array}$ & $\begin{array}{c}4^{\text {th }} \text { F-up } \\
120^{\text {th }} \text { day }\end{array}$ & $\begin{array}{c}5^{\text {th }} \text { F-up } \\
150^{\text {th }} \text { day }\end{array}$ & $\begin{array}{c}6^{\text {th }} \text { F-up } \\
180^{\text {th }} \text { day }\end{array}$ & $\begin{array}{c}7^{\text {th }} \text { F-up } \\
210^{\text {th }} \text { day }\end{array}$ & $\begin{array}{l}8^{\text {th }} \text { F-up } \\
240^{\text {th }} \text { day }\end{array}$ \\
\hline SGOT (IU/L) & $\begin{array}{l}25.75 \\
\pm 7.70 \\
\end{array}$ & $\begin{array}{c}35.44 \\
\pm 64.87 \\
\end{array}$ & $\begin{array}{r}27.13 \\
\pm 17.52 \\
\end{array}$ & $\begin{array}{l}25.72 \\
\pm 9.72 \\
\end{array}$ & $\begin{array}{r}39.95 \\
\pm 29.01 \\
\end{array}$ & $\begin{array}{l}25.91 \\
\pm 0.28 \\
\end{array}$ & $\begin{array}{c}24.9 \\
\pm 12.86 \\
\end{array}$ & $\begin{array}{c}24.44 \\
\pm 10.99 \\
\end{array}$ & $\begin{array}{l}23.38 \\
\pm 9.10 \\
\end{array}$ \\
\hline SGPT (IU/L) & $\begin{array}{c}23.72 \\
\pm 12.91 \\
\end{array}$ & $\begin{array}{r}33.26 \\
\pm 66.23 \\
\end{array}$ & $\begin{array}{r}31.09 \\
\pm 55.6 \\
\end{array}$ & $\begin{array}{r}24.60 \\
\pm 11.56 \\
\end{array}$ & $\begin{array}{c}26.02 \\
\pm 21.51 \\
\end{array}$ & $\begin{array}{r}26.05 \\
\pm 24.48 \\
\end{array}$ & $\begin{array}{c}26.16 \\
\pm 19.39 \\
\end{array}$ & $\begin{array}{r}23.79 \\
\pm 22.3 \\
\end{array}$ & $\begin{array}{r}23.73 \\
\pm 15.97 \\
\end{array}$ \\
\hline $\begin{array}{l}\begin{array}{c}\text { Bilirubin } \\
(\mathrm{mg} / \mathrm{dl})\end{array} \\
\end{array}$ & $\begin{array}{c}0.76 \\
\pm 0.21 \\
\end{array}$ & $\begin{array}{c}0.81 \\
\pm 0.26 \\
\end{array}$ & $\begin{array}{c}0.78 \\
\pm 0.22 \\
\end{array}$ & $\begin{array}{c}0.79 \\
\pm 0.44 \\
\end{array}$ & $\begin{array}{c}0.78 \\
\pm 0.24 \\
\end{array}$ & $\begin{array}{c}0.75 \\
\pm 0.21 \\
\end{array}$ & $\begin{array}{c}0.78 \\
\pm 0.24 \\
\end{array}$ & $\begin{array}{c}0.77 \pm 0 \\
.21\end{array}$ & $\begin{array}{c}0.76 \\
\pm 0.23 \\
\end{array}$ \\
\hline $\begin{array}{l}\text { Blood Urea } \\
\text { (mg/dl) }\end{array}$ & $\begin{array}{r}23.33 \\
\pm 5.75 \\
\end{array}$ & $\begin{array}{l}22.12 \\
\pm 5.91 \\
\end{array}$ & $\begin{array}{l}23.58 \\
\pm 8.43 \\
\end{array}$ & $\begin{array}{l}23.05 \\
\pm 7.87 \\
\end{array}$ & $\begin{array}{l}22.68 \\
\pm 6.42 \\
\end{array}$ & $\begin{array}{r}23.36 \\
\pm 7.23 \\
\end{array}$ & $\begin{array}{l}21.44 \\
\pm 6.41 \\
\end{array}$ & $\begin{array}{r}21.38 \\
\pm 5.80 \\
\end{array}$ & $\begin{array}{l}23.18 \\
\pm 5.50 \\
\end{array}$ \\
\hline $\begin{array}{l}\text { Creatinine } \\
(\mathrm{mg} / \mathrm{dl})\end{array}$ & $\begin{array}{c}0.89 \\
\pm 0.14\end{array}$ & $\begin{array}{c}0.91 \\
\pm 0.15\end{array}$ & $\begin{array}{c}0.92 \\
\pm 0.16\end{array}$ & $\begin{array}{c}0.92 \\
\pm 0.17\end{array}$ & $\begin{array}{c}0.91 \\
\pm 0.17\end{array}$ & $\begin{array}{c}0.93 \\
\pm 0.14\end{array}$ & $\begin{array}{c}0.93 \\
\pm 0.17\end{array}$ & $\begin{array}{c}0.92 \\
\pm 0.16\end{array}$ & $\begin{array}{c}0.94 \\
\pm 0.18\end{array}$ \\
\hline $\begin{array}{l}\text { Protein } \\
(\mathrm{gm} / 100 \mathrm{ml})\end{array}$ & $\begin{array}{c}7.04 \\
\pm 0.45\end{array}$ & $\begin{array}{c}6.92 \\
\pm 0.55\end{array}$ & $\begin{array}{r}7.08 \\
\pm 0.51\end{array}$ & $\begin{array}{c}6.97 \\
\pm 0.50\end{array}$ & $\begin{array}{c}7.12 \\
\pm 0.48\end{array}$ & $\begin{array}{r}7.20 \\
\pm 0.59\end{array}$ & $\begin{array}{r}7.20 \\
\pm 0.52\end{array}$ & $\begin{array}{c}7.21 \\
\pm 0.52\end{array}$ & $\begin{array}{c}7.07 \\
\pm 0.52\end{array}$ \\
\hline $\begin{array}{c}\text { Albumin } \\
\text { (gm/100ml) }\end{array}$ & $\begin{array}{c}3.98 \\
\pm 0.42\end{array}$ & $\begin{array}{c}3.96 \\
\pm 0.38\end{array}$ & $\begin{array}{c}3.85 \\
\pm 0.38\end{array}$ & $\begin{array}{r}3.89 \\
\pm 0.45 \\
\end{array}$ & $\begin{array}{c}3.88 \\
\pm 0.50\end{array}$ & $\begin{array}{c}3.80 \\
\pm 0.48\end{array}$ & $\begin{array}{c}3.91 \\
\pm 0.43\end{array}$ & $\begin{array}{r}3.80 \\
\pm 0.45\end{array}$ & $\begin{array}{c}3.85 \\
\pm 0.44 \\
\end{array}$ \\
\hline $\begin{array}{l}\text { Globulin } \\
\text { (gm/100ml) }\end{array}$ & $\begin{array}{c}3.09 \\
\pm 0.57\end{array}$ & $\begin{array}{c}3.02 \\
\pm 0.52 \\
\end{array}$ & $\begin{array}{c}3.24 \\
\pm 0.62\end{array}$ & $\begin{array}{r}3.03 \\
\pm 0.62\end{array}$ & $\begin{array}{c}3.21 \\
\pm 0.61\end{array}$ & $\begin{array}{c}3.34 \\
\pm 0.56\end{array}$ & $\begin{array}{c}3.27 \\
\pm 0.58\end{array}$ & $\begin{array}{c}3.37 \\
\pm 0.59\end{array}$ & $\begin{array}{r}3.20 \\
\pm 0.49\end{array}$ \\
\hline A/ G Ratio & $\begin{array}{c}1.34 \\
\pm 0.37 \\
\end{array}$ & $\begin{array}{c}1.38 \\
\pm 0.36 \\
\end{array}$ & $\begin{array}{c}1.24 \\
\pm 0.37 \\
\end{array}$ & $\begin{array}{r}1.33 \\
\pm 0.45 \\
\end{array}$ & $\begin{array}{r}1.26 \\
\pm 0.44 \\
\end{array}$ & $\begin{array}{c}1.17 \\
\pm 0.31 \\
\end{array}$ & $\begin{array}{c}1.22 \\
\pm 0.35 \\
\end{array}$ & $\begin{array}{r}1.15 \\
\pm 0.34 \\
\end{array}$ & $\begin{array}{c}1.21 \\
\pm 0.29 \\
\end{array}$ \\
\hline
\end{tabular}


Table 3.Effect of allopathic drug Melanocyl Tablet (Methoxsalen) on the levels of SGPT, SGOT, Bilirubin, Blood Urea and Serum Creatinine, Protein, Albumin, Globulin and A/ G ratio in vitiligo patients

\begin{tabular}{|c|c|c|c|c|c|c|c|c|c|}
\hline $\begin{array}{c}\text { Group } \\
\text { Parameter }\end{array}$ & $\begin{array}{c}\text { BL } \\
\text { 0-Day }\end{array}$ & $\begin{array}{l}1^{\text {st }} F-U p \\
30^{\text {th }} \text { day }\end{array}$ & $\begin{array}{l}2^{\text {nd }} \text { F-up } \\
60^{\text {th }} \text { day }\end{array}$ & \begin{tabular}{|l|}
$3^{\text {rd }}$ F-up \\
$90^{\text {th }}$ day
\end{tabular} & $\begin{array}{c}4^{\text {th }} \text { F-up } \\
120^{\text {th }} \text { day }\end{array}$ & $\begin{array}{l}5^{\text {th }} \text { F-up } \\
150^{\text {th }} \text { day }\end{array}$ & $\begin{array}{c}6^{\text {th }} \text { F-up } \\
180^{\text {th }} \text { day }\end{array}$ & $\begin{array}{c}7^{\text {th }} \text { F-up } \\
210^{\text {th }} \text { day }\end{array}$ & $\begin{array}{l}8^{\text {th }} \text { F-up } \\
240^{\text {th }} \text { day }\end{array}$ \\
\hline SGOT (IU/L) & $\begin{array}{l}24.20 \\
\pm 8.35 \\
\end{array}$ & $\begin{array}{l}25.54 \\
\pm 8.29 \\
\end{array}$ & $\begin{array}{c}26.29 \\
\pm 10.02 \\
\end{array}$ & $\begin{array}{r}24.3 \\
\pm 8.97 \\
\end{array}$ & $\begin{array}{l}24.85 \\
\pm 9.04 \\
\end{array}$ & $\begin{array}{r}23.69 \\
\pm 10.98 \\
\end{array}$ & $\begin{array}{r}23.78 \\
\pm 12.00 \\
\end{array}$ & $\begin{array}{c}24.21 \\
\pm 12.03 \\
\end{array}$ & $\begin{array}{r}23.1 \\
\pm 7.89 \\
\end{array}$ \\
\hline SGPT (IU/L) & $\begin{array}{c}25.04 \\
\pm 13.13 \\
\end{array}$ & $\begin{array}{c}24.9 \\
\pm 10.79 \\
\end{array}$ & $\begin{array}{c}24.04 \\
\pm 15.34 \\
\end{array}$ & $\begin{array}{c}24.97 \\
\pm 14.19\end{array}$ & $\begin{array}{c}23.39 \\
\pm 21.51 \\
\end{array}$ & $\begin{array}{c}24.18 \\
\pm 24.48 \\
\end{array}$ & $\begin{array}{c}23.91 \\
\pm 19.39 \\
\end{array}$ & $\begin{array}{l}22.48 \\
\pm 22.3 \\
\end{array}$ & $\begin{array}{r}20.40 \\
\pm 15.97 \\
\end{array}$ \\
\hline $\begin{array}{c}\text { Bilirubin } \\
(\mathrm{mg} / \mathrm{dl})\end{array}$ & $\begin{array}{c}0.73 \\
\pm 0.22 \\
\end{array}$ & $\begin{array}{c}0.71 \\
\pm 0.22 \\
\end{array}$ & $\begin{array}{r}1.09 \\
\pm 2.97 \\
\end{array}$ & $\begin{array}{r}0.69 \\
\pm 0.22 \\
\end{array}$ & $\begin{array}{c}1.11 \\
\pm 3.16 \\
\end{array}$ & $\begin{array}{c}0.74 \\
\pm 0.25 \\
\end{array}$ & $\begin{array}{c}0.70 \\
\pm 0.23 \\
\end{array}$ & $\begin{array}{c}0.71 \\
\pm 0.22 \\
\end{array}$ & $\begin{array}{c}0.71 \\
\pm 0.24 \\
\end{array}$ \\
\hline $\begin{array}{l}\text { Blood Urea } \\
\text { (mg/dl) }\end{array}$ & $\begin{array}{l}21.74 \\
\pm 6.47\end{array}$ & $\begin{array}{l}21.49 \\
\pm 6.34 \\
\end{array}$ & $\begin{array}{l}22.09 \\
\pm 7.76 \\
\end{array}$ & $\begin{array}{l}23.01 \\
\pm 6.92 \\
\end{array}$ & $\begin{array}{l}21.14 \\
\pm 6.66 \\
\end{array}$ & $\begin{array}{l}21.95 \\
\pm 8.19 \\
\end{array}$ & $\begin{array}{l}21.14 \\
\pm 6.22\end{array}$ & $\begin{array}{l}22.03 \\
\pm 6.26 \\
\end{array}$ & $\begin{array}{l}23.16 \\
\pm 6.88 \\
\end{array}$ \\
\hline $\begin{array}{c}\text { Creatinine } \\
(\mathrm{mg} / \mathrm{dl})\end{array}$ & $\begin{array}{c}0.90 \\
\pm 0.16 \\
\end{array}$ & $\begin{array}{c}0.91 \\
\pm 0.16 \\
\end{array}$ & $\begin{array}{r}0.86 \\
\pm 0.15 \\
\end{array}$ & $\begin{array}{r}0.90 \\
\pm 0.15 \\
\end{array}$ & $\begin{array}{c}0.88 \\
\pm 0.16 \\
\end{array}$ & $\begin{array}{c}0.92 \\
\pm 0.13 \\
\end{array}$ & $\begin{array}{c}0.87 \\
\pm 0.15 \\
\end{array}$ & $\begin{array}{r}0.90 \\
\pm 0.16 \\
\end{array}$ & $\begin{array}{c}0.92 \\
\pm 0.17 \\
\end{array}$ \\
\hline $\begin{array}{c}\text { Protein } \\
(\mathrm{gm} / 100 \mathrm{ml})\end{array}$ & $\begin{array}{c}7.03 \\
\pm 0.55\end{array}$ & $\begin{array}{c}7.08 \\
\pm 0.55\end{array}$ & $\begin{array}{c}7.14 \\
\pm 0.56\end{array}$ & $\begin{array}{c}7.15 \\
\pm 0.68\end{array}$ & $\begin{array}{c}7.14 \\
\pm 0.61\end{array}$ & $\begin{array}{c}7.01 \\
\pm 0.51\end{array}$ & $\begin{array}{c}7.17 \\
\pm 0.53\end{array}$ & $\begin{array}{c}7.06 \\
\pm 0.50\end{array}$ & $\begin{array}{c}7.16 \\
\pm 0.53\end{array}$ \\
\hline $\begin{array}{l}\text { Albumin } \\
\text { (gm/100ml) }\end{array}$ & $\begin{array}{c}4.00 \\
\pm 0.43\end{array}$ & $\begin{array}{c}4.03 \\
\pm 0.42\end{array}$ & $\begin{array}{c}3.87 \\
\pm 0.37\end{array}$ & $\begin{array}{c}3.92 \\
\pm 0.48\end{array}$ & $\begin{array}{c}3.86 \\
\pm 0.50\end{array}$ & $\begin{array}{c}3.84 \\
\pm 0.44\end{array}$ & $\begin{array}{c}3.91 \\
\pm 0.44\end{array}$ & $\begin{array}{c}3.88 \\
\pm 0.38\end{array}$ & $\begin{array}{c}3.95 \\
\pm 0.80\end{array}$ \\
\hline $\begin{array}{c}\text { Globulin } \\
\text { (gm/100ml) }\end{array}$ & $\begin{array}{c}3.06 \\
\pm 0.68\end{array}$ & $\begin{array}{c}3.04 \\
\pm 0.59\end{array}$ & $\begin{array}{c}3.27 \\
\pm 0.60\end{array}$ & $\begin{array}{c}3.24 \\
\pm 0.63\end{array}$ & $\begin{array}{c}3.26 \\
\pm 0.66\end{array}$ & $\begin{array}{c}3.17 \\
\pm 0.55\end{array}$ & $\begin{array}{c}3.26 \\
\pm 0.58 \\
\end{array}$ & $\begin{array}{c}3.19 \\
\pm 0.51\end{array}$ & $\begin{array}{c}3.25 \\
\pm 0.59\end{array}$ \\
\hline A/ G Ratio & $\begin{array}{c}1.37 \\
\pm 0.38\end{array}$ & $\begin{array}{c}1.37 \\
\pm 0.38\end{array}$ & $\begin{array}{c}1.22 \\
\pm 0.35\end{array}$ & $\begin{array}{c}1.27 \\
\pm 0.39\end{array}$ & $\begin{array}{c}1.23 \\
\pm 0.37\end{array}$ & $\begin{array}{c}1.22 \\
\pm 0.30\end{array}$ & $\begin{array}{r}1.23 \\
\pm 0.35\end{array}$ & $\begin{array}{c}1.24 \\
\pm 0.28\end{array}$ & $\begin{array}{c}1.25 \\
\pm 0.26\end{array}$ \\
\hline
\end{tabular}

Table 4.Effect of Unani coded drug UNIM-00I + UNIM-003 in the levels of Haemoglobin, R.B.C. Count, Total Leukocyte Count (T.L.C.), Erythrocyte Sedimentation Rate (E.S.R.), Polymorph, Lymphocyte and Eosinophil count in Vitiligo patients

\begin{tabular}{|c|c|c|c|c|c|c|c|c|c|}
\hline \multirow{2}{*}{$\begin{array}{c}\text { Group } \\
\text { Parameter }\end{array}$} & \multirow{2}{*}{$\begin{array}{l}\text { Haemoglo- } \\
\text { bin (gm \%) }\end{array}$} & \multirow{2}{*}{$\begin{array}{c}\text { R.B.C. } \\
(106 \mathrm{~mm})\end{array}$} & \multirow{2}{*}{$\begin{array}{c}\text { T.L.C. } \\
(103 / \mathrm{mm} 3)\end{array}$} & \multicolumn{2}{|c|}{$\mathrm{ESR}(\mathrm{mm} / \mathrm{hr})$} & \multirow{2}{*}{$\begin{array}{c}\text { Platelets } \\
\text { (Lac/mm3) }\end{array}$} & \multirow{2}{*}{$\begin{array}{l}\text { Polymo- } \\
\text { rphs (\%) }\end{array}$} & \multirow{2}{*}{$\begin{array}{c}\text { Lymph- } \\
\text { ocyte (\%) }\end{array}$} & \multirow{2}{*}{$\begin{array}{l}\text { Eosino- } \\
\text { phil (\%) }\end{array}$} \\
\hline & & & & $1^{\text {st }} \mathrm{hrs}$ & $2^{\text {nd }}$ hrs & & & & \\
\hline $\mathrm{BL}(0$ & 12.5 & $4.16 \pm 0$ & $7.91 \pm 1.9$ & & $\begin{array}{c}42.0 \\
\pm 13.23\end{array}$ & $2.11 \pm 0.64$ & & $\begin{array}{c}34.0 \\
\pm 8.50\end{array}$ & $\begin{array}{c}4.0 \\
\pm 2.03\end{array}$ \\
\hline $\begin{array}{c}1^{\text {st }} \mathrm{F}-\text { Up } \\
\left(30^{\text {th }} \text {-days }\right)\end{array}$ & $11.99 \pm 2.22$ & $4.01 \pm 0.72$ & $7.85 \pm 1.71$ & & & 01 & & & $\begin{array}{r}5.0 \\
\pm 7.41 \\
\end{array}$ \\
\hline $\begin{array}{c}2^{\text {nd }} \text { F-up (60- } \\
\text { Days) }\end{array}$ & .81 & $4.09 \pm c$ & 7.75 & & $\begin{array}{c}44.0 \\
\pm 11.99 \\
\end{array}$ & 7 & & & $\begin{array}{c}6.0 \\
\pm 2.54 \\
\end{array}$ \\
\hline $\begin{array}{c}3^{\text {rd }} \mathrm{F} \text {-up } \\
\left(90^{\text {th }} \text {-days }\right)\end{array}$ & $12.33 \pm 1.90$ & $4.12 \pm 0.66$ & $7.68 \pm$ & & $\begin{array}{c}42.0 \\
\pm 12.81 \\
\end{array}$ & 0 & & & $\begin{array}{c}4.0 \\
\pm 2.28\end{array}$ \\
\hline $\begin{array}{c}4^{\text {th }} \text { F-up } \\
\left(120^{\text {th }} \text {-days }\right)\end{array}$ & $12.20+1.77$ & $4.06 \pm 0$ & $7.68 \pm$ & & & $212+$ & & & \\
\hline $\begin{array}{c}5^{\text {th }} \text { F-up } \\
\left(150^{\text {th }} \text {-days }\right)\end{array}$ & $12.37 \pm 1.85$ & $4.12 \pm 0.60$ & $7.60 \pm 1.75$ & $\begin{array}{l}31.0 \pm \\
14.19\end{array}$ & $\begin{array}{c}42.0 \\
\pm 13.47\end{array}$ & $2.11 \pm 0.54$ & & & $\begin{array}{c}5.0 \\
\pm 2.43\end{array}$ \\
\hline $\begin{array}{c}6^{\text {th }} \text { F-up } \\
\left(180^{\text {th }} \text {-days }\right)\end{array}$ & $12.47 \pm 1.81$ & $4.16 \pm 0.59$ & $7.55 \pm 1.55$ & $\begin{array}{l}32.0 \pm \\
12.65 \\
\end{array}$ & $\begin{array}{c}43.0 \\
\pm 11.57 \\
\end{array}$ & $2.14 \pm 0.57$ & $\begin{array}{c}63.0 \\
\pm 10.27 \\
\end{array}$ & $\begin{array}{r}32.0 \\
\pm 9.42 \\
\end{array}$ & $\begin{array}{r}5.0 \\
\pm 2.38 \\
\end{array}$ \\
\hline $\begin{array}{c}7^{\text {th }} \text { F-up } \\
\left(210^{\text {th }} \text {-days }\right)\end{array}$ & $12.62 \pm 1.80$ & $4.22 \pm 0.61$ & $7.66 \pm 1.91$ & $\begin{array}{l}30.0 \pm \\
14.78 \\
\end{array}$ & $\begin{array}{c}41.0 \\
\pm 13.91 \\
\end{array}$ & $2.09 \pm 0.66$ & $\begin{array}{r}63.0 \\
\pm 9.70 \\
\end{array}$ & $\begin{array}{r}32.0 \\
\pm 8.83 \\
\end{array}$ & $\begin{array}{c}5.0 \\
\pm 2.06 \\
\end{array}$ \\
\hline $\begin{array}{c}8^{\text {th }} \text { F-up } \\
\left(240^{\text {th }} \text {-days }\right)\end{array}$ & $12.40 \pm 1.77$ & $4.22 \pm 0.59$ & $7.42 \pm 1.3$ & $\begin{array}{l}30.0 \pm \\
13.56\end{array}$ & $\begin{array}{c}42.0 \\
\pm 13.17\end{array}$ & $08 \pm 0.63$ & $\begin{array}{r}64.0 \\
\pm 9.08\end{array}$ & $\begin{array}{c}31.0 \\
\pm 8.32\end{array}$ & $\begin{array}{c}5.0 \\
\pm 2.37\end{array}$ \\
\hline
\end{tabular}




\section{Haematological Studies}

In both trial and control group, no significant alterations in the level of hemoglobin, Red Blood Corpuscles (RBC), Total Leucocytes Counts (TLC), Erythrocyte Sedimentation Rate (ESR) and Differential Leucocytes Counts (DLC) had been observed. When compared with the values of baseline and different follow-up of treatment (Table 4 and 5). Similar observation had reported by other authors. ${ }^{53,54,55}$

\section{Repigmentation Response}

In trial group out 65 patients studied, 32 patients showed 0-20\% Repigmentation, 27 patients showed 21-70\% Repigmentation and 06 patients showed 71$100 \%$ Repigmentation. In control group out 67 patients studied, 36 patients showed 0-20\% Repigmentation, 23 patients showed $21-70 \%$ Repigmentation and 08 patients showed $71-100 \%$ Repigmentation (Table 6). Nazim et al., $2018^{56}$ had reported the safety and efficacy of many
Unani drugs with different percentage of Repigmentation.

\section{Conclusion}

In modern medicine system various therapy such as topical corticosteroids, systemic drug, ultraviolet light therapy, Monochromatic Excimer Light laser (MEL) therapy, Combination of UV have recently been used for treatment of Vitiligo, but they all have certain side effects. Thus there is an urgent demand to identify herbal drugs and its active constituents, used as potential therapeutic agents for treatment of Vitiligo (bars).

On the basis of this study, it may be concluded that UNIM-001 Tablet and UNIM-003 topical drug. As well as comparator Drug (Melanocyl Tablet (Methoxsalen)) Possesses anti-Vitiligo effect. It can also be inferred that both the drug are safe as they did not induce any toxic effect, particularly on liver and kidney functions. Further studies are warranted in large group.

Table 5.Effect of Melanocyl tablets (methoxsalen) in the levels of Haemoglobin, R.B.C. Count, Total Leukocyte Count (T.L.C.), Erythrocyte Sedimentation Rate (E.S.R.), Polymorph, Lymphocyte and Eosinophil count in Vitiligo patients

\begin{tabular}{|c|c|c|c|c|c|c|c|c|c|}
\hline $\begin{array}{c}\text { Group } \\
\text { Parameter }\end{array}$ & $\begin{array}{l}\text { Haemoglo- } \\
\text { bin (gm \%) }\end{array}$ & $\begin{array}{l}\text { R.B.C. } \\
\text { (106/ } \\
\mathrm{mm} 3)\end{array}$ & $\begin{array}{l}\text { T.L.C. } \\
(103 / \\
\mathrm{mm} 3)\end{array}$ & $\begin{array}{c}\text { ESR (mm/ } \\
\text { hr) } 1^{\text {st }} \mathrm{hrs} \\
2^{\text {nd }} \mathrm{hrs}\end{array}$ & $\begin{array}{c}\text { Platelets } \\
\text { (Lac/ } \\
\mathrm{mm} 3 \text { ) }\end{array}$ & $\begin{array}{l}\text { Polymo- } \\
\text { rphs (\%) }\end{array}$ & $\begin{array}{l}\text { Lymph- } \\
\text { ocyte (\%) }\end{array}$ & $\begin{array}{l}\text { Eosino- } \\
\text { phil (\%) }\end{array}$ & $\begin{array}{l}\text { Group } \\
\text { Parameter }\end{array}$ \\
\hline BL (0-Day) & $12.14 \pm 1.72$ & & & & & & & & $4.0 \pm 2.30$ \\
\hline $\begin{array}{c}1^{\text {st }} \mathrm{F}-\text { Up } \\
\left(30^{\text {th }} \text {-days }\right)\end{array}$ & $12.01 \pm 1.81$ & & & & & & & & $4.0 \pm 2.38$ \\
\hline $\begin{array}{c}2^{\text {nd }} \text { F-up } \\
\left(60^{\text {th }} \text {-Days }\right)\end{array}$ & $12.00 \pm 1.86$ & $\begin{array}{c}4.03 \\
\pm 0.61\end{array}$ & $\begin{array}{c}7.70 \\
\pm 2.00\end{array}$ & $\begin{array}{c}30.0 \\
\pm 13.37\end{array}$ & & & & & $5.0 \pm 2.46$ \\
\hline $\begin{array}{c}3^{\text {rd }} \mathrm{F}-\text { up } \\
\left(90^{\text {th }} \text {-days }\right)\end{array}$ & $1.96 \pm 1.64$ & $\begin{array}{c}4.03 \\
\pm 0.59 \\
\end{array}$ & & & & & & & $4.0 \pm 2.05$ \\
\hline $\begin{array}{c}4^{\text {th }} \text { F-up } \\
\left(120^{\text {th}} \text {-days }\right)\end{array}$ & $12.09 \pm 1.67$ & & & & & & & & $4.0 \pm 1.95$ \\
\hline $\begin{array}{c}5^{\text {th }} \text { F-up } \\
\left(150^{\text {th }} \text {-days }\right)\end{array}$ & $12.10 \pm 1.73$ & $\begin{array}{c}4.08 \\
\pm 0.56\end{array}$ & $\begin{array}{c}7.16 \\
\pm 1.60\end{array}$ & $\begin{array}{c}31.0 \\
\pm 14.46\end{array}$ & $\begin{array}{c}42.0 \\
\pm 13.35\end{array}$ & $\begin{array}{c}2.15 \\
\pm 0.70\end{array}$ & & & $5.0 \pm 2.55$ \\
\hline $\begin{array}{c}6^{\text {th }} \mathrm{F}-\text { up } \\
\left(180^{\text {th }} \text {-days }\right)\end{array}$ & $12.12 \pm 1.72$ & $\begin{array}{c}4.04 \\
\pm 0.51\end{array}$ & $\begin{array}{c}7.42 \\
\pm 2.13\end{array}$ & $\begin{array}{c}31.0 \\
\pm 14.46\end{array}$ & $\begin{array}{c}42.0 \\
\pm 13.35\end{array}$ & $\begin{array}{c}2.15 \\
\pm 0.70\end{array}$ & $\begin{array}{c}61.0 \\
\pm 9.27 \\
\end{array}$ & $\begin{array}{c}34.0 \\
\pm 9.51\end{array}$ & $5.0 \pm 2.55$ \\
\hline $\begin{array}{c}7^{\text {th }} \text { F-up } \\
\left(210^{\text {th }} \text {-days }\right)\end{array}$ & $12.14 \pm 1.70$ & $\begin{array}{c}4.09 \\
\pm 0.55 \\
\end{array}$ & $\begin{array}{r}7.39 \\
\pm 1.88 \\
\end{array}$ & $\begin{array}{c}30.0 \\
\pm 13.98 \\
\end{array}$ & $\begin{array}{c}41.0 \\
\pm 13.29 \\
\end{array}$ & $\begin{array}{c}2.14 \\
\pm 0.76 \\
\end{array}$ & $\begin{array}{r}63.0 \\
\pm 9.93 \\
\end{array}$ & $\begin{array}{r}33.0 \\
\pm 9.69 \\
\end{array}$ & $4.0 \pm 2.39$ \\
\hline $\begin{array}{c}8^{\text {th }} \text { F-up } \\
\left(240^{\text {th }} \text {-days }\right)\end{array}$ & $12.22 \pm 1 . \varepsilon$ & $\begin{array}{c}4.14 \\
\pm 0.61\end{array}$ & $\begin{array}{r}7.41 \\
\pm 1.65 \\
\end{array}$ & $\begin{array}{c}30.0 \\
\pm 13.49\end{array}$ & $\begin{array}{c}42.0 \\
\pm 12.39\end{array}$ & $\begin{array}{c}2.19 \\
\pm 0.68\end{array}$ & $\begin{array}{r}61.0 \\
\pm 9.53 \\
\end{array}$ & $\begin{array}{r}34.0 \\
\pm 9.86 \\
\end{array}$ & 4 \\
\hline
\end{tabular}

Table 6.Effect of Unani coded drug UNIM-00I + UNIM-003 (Trial Group) and allopathic drug Melanocyl Tablet (Methoxsalen) (Control group) on percentage Repigmentation after post-treatment (240-days) in Vitiligo patients

\begin{tabular}{|c|c|c|}
\hline Pigmentation in \%age & Trial Group $(\mathbf{n}=\mathbf{6 5})$ & Control Group $(\mathbf{n}=\mathbf{6 7})$ \\
\hline $0-20 \%$ (No response) & 32 & 36 \\
\hline $21-70 \%$ (Partial Response) & 27 & 23 \\
\hline $71-100 \%$ (Response) & 06 & 08 \\
\hline
\end{tabular}




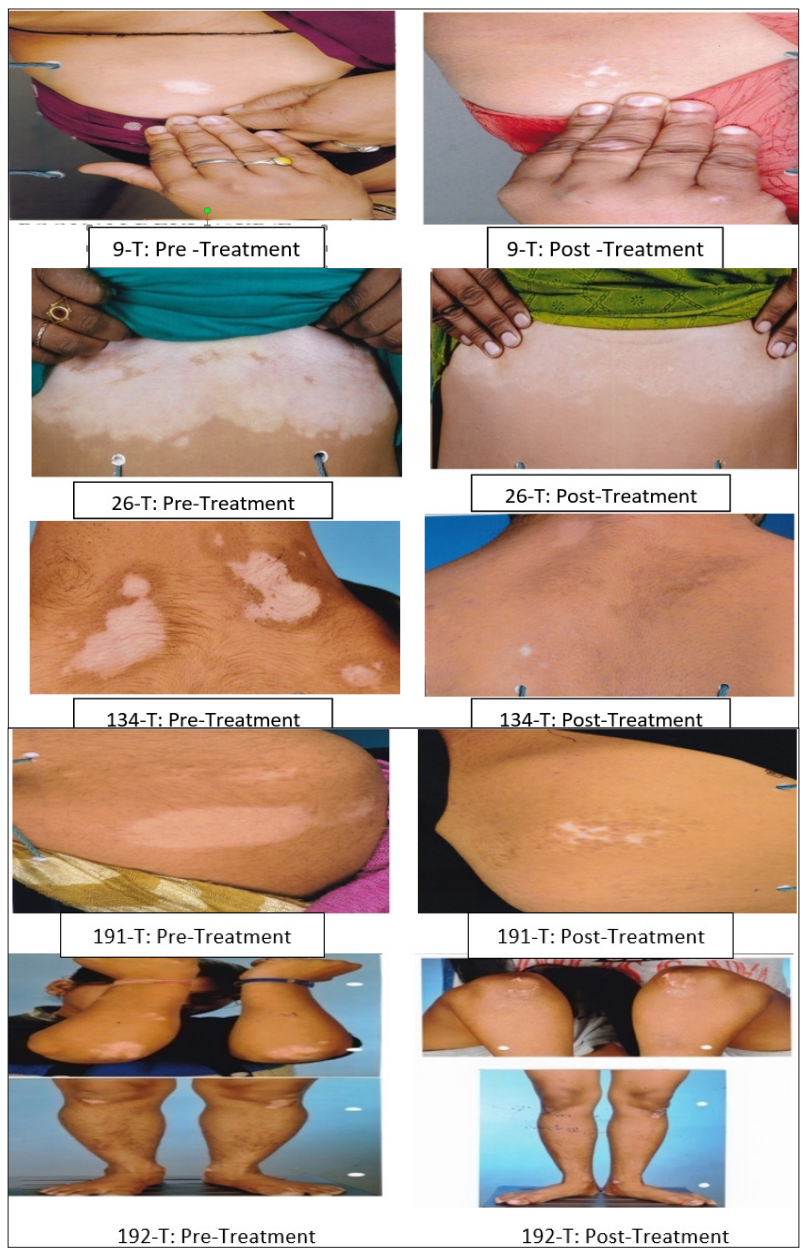

Figure I.Photographs of trial group showing response to Unani coded drugs UNIM-00I Tablet and UNIM-003 topical in vitiligo patients. (pre-treatment $=0$-day, post-treatment $=240$-days)

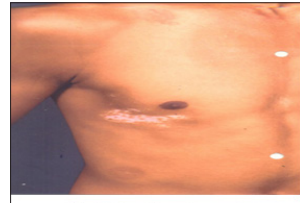

92-C: Pre-Treatment

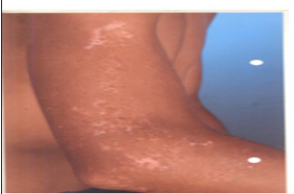

92-C: Pre-Treatment

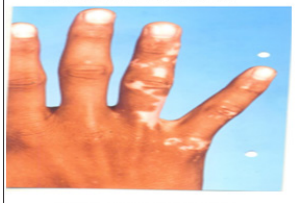

92-C: Pre-Treatment

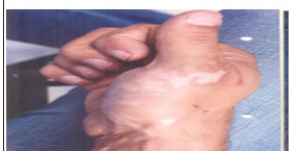

85-C: Pre-Treatment

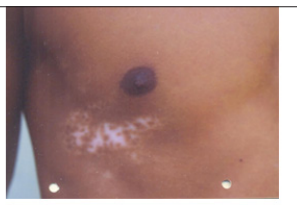

92-C: Post-Treatment

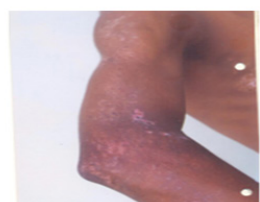

(2) $r \cdot D$ ct Trantmont

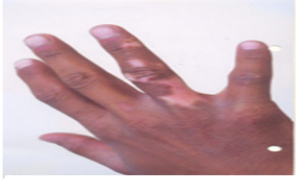

92-C:Post-Treatment

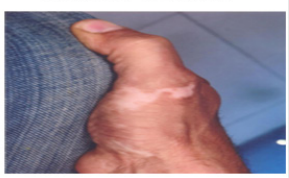

85-C: Post-Treatment

Figure 2.Photographs of Control group showing response of Melanocyl (Methoxsalen) Tablet in vitiligo patients. pre-treatment $=0$-day, post-treatment $=240$-days 


\section{Acknowledgement}

The authors are indebted to Central Council for Research in Unani Medicine, New Delhi for encouragement, guidance and financial support. We thank Mr Kushal Pal Singh, Mr Javed Akther, Mr Mohd Akbar Rais, Mr Tariq Ali Beg Lab Technicians and Mr Shish Mohammad, Lab Attendant of Biochemistry and Pathology Laboratory RRIUM, Aligarh for investigations.

\section{Conflict of Interest: None}

\section{References}

1. Ali KA, Felsten LM, Daly M, Petronic-Rosic V, Vitiligo. A comprehensive overview part I. Introduction, epidemiology, quality of life, diagnosis, differential diagnosis, associations, histopathology, etiology and work-up. J Am Acad Dermatol 2011; 65: 473.

2. Krüger $\mathrm{C}$, Schallreuter $\mathrm{KU}$. A review of the worldwide prevalence of vitiligo in children/adolescents and adults. Int J Dermatol 2012; 51: 1206.

3. Behl PN, Aggarwal A, Srivastava G, Vitiligo. In Practice of Dermatology: Ed. by PN Behl, G Srivastava (Eds.), 9th (Edn.) (CBS Publishers: New Delhi), 2003, 238.

4. Srivastava G, Vitiligo-Introduction Asian Clinic. Dermatol, 1(1978) 1.

5. Vora RV, Patel BB, Chaudhary AH, Mehta MJ, Pilani AP. A clinical study of vitiligo in a rural set up of Gujarat. Indian J Community Med 2014; 39: 143.

6. Mahajan VK, Vashist S, Chauhan PS, Mehta KIS, Sharma V, Sharma A. Clinico-epidemiological profile of patients with vitiligo: A retrospective study from a tertiary care center of North India. Indian Dermatol Online J, 2019; 10(1): 38.

7. Sams WM, Lynch PJ. Principles and practice of dermatology [Internet]. (Churchill Livingstone) 1990, 1014.

8. Taıeb A, Picardo M. "Vitiligo," The New England Journal of Medicine 2009; 360(2): 160.

9. Thierry P, Jean-Paul O. Physiopathology and genetics of vitiligo. Journal of Autoimmunity 2005; 25: 63.

10. Nooshin B. The Newest Hypothesis about Vitiligo: Most of the Suggested Pathogeneses of Vitiligo Can Be Attributed to Lack of One Factor, Zinc- $\alpha 2-G l y c o p r o t e i n$. ISRN Dermatology 2012; (2012) 1.

11. Banerjee N, Gayen S, Modak D, Sarkar S, Saha B, Mukhopadhyay S. Systemic redox imbalance along with increased serum sialic acid is prevalent in patients with active vitiligo: A study from a tertiary care teaching hospital of eastern India. Indian J Dermatol 2019; 64(2): 97.

12. Steven J G. Vitiligo, reactive oxygen species and T-cells. Clin Sci (Lond), 2011; 120(3): 99.

13. Cucchi ML, Frattini P, Santagostino G, Preda S, Orecchia G. Catecholamines increase in the urine of non-segmental vitiligo especially during its active phase. Pigment Cell Res 2003; 16(2): 111.

14. Roselyne YW, Flavie L, Muriel Cario-André, Alain R, Valérie P, Benzekri L et al. Altered E-Cadherin Levels and Distribution in Melanocytes Precede Clinical Manifestations of Vitiligo. Journal of Investigative Dermatology 2015; 135(7): 1810.

15. Boniface K, Seneschal J, Picardo M, Taïeb A,.Vitiligo: Focus on Clinical Aspects, Immunopathogenesis, and Therapy. Clin Rev Allergy Immunol 2018; 54: 52.

16. Esmat S, Hegazy RA, Shalaby S, Chu-Sung Hu S, Lan CCE. Phototherapy and Combination Therapies for Vitiligo. Dermatol Clin 2017; 35: 171.

17. Zhang Y, Cai Y, Shi M, Jiang S, Cui S, Wu Y et al. The Prevalence of Vitiligo: A Meta-Analysis. PLoS ONE 2016; 11(9): 1.

18. Njoo MD, Westerhof W, Bos JD, Bossuyt PMM. The development of guidelines for the treatment of vitiligo. Arch Dermatol 1999; 135: 1514.

19. Schaffer JV, Bolognia JL. The treatment of hypopigmentation in children. Clin Dermatol 2003; 21(4): 296.

20. Kostovic K, Pasic A. New treatment modalities for vitiligo. Drugs 2005; 65(4): 447.

21. Köse $O$, Arca E, Kurumlu Z. Mometasone cream versus pimecrolimus cream for the treatment of childhood localized vitiligo. J Dermatolog Treat 2010; 21: 133.

22. Singh $H$, Kumaran MS, Bains A, Parsad D. A randomized comparative study of oral corticosteroid minipulse and low-dose oral methotrexate in the treatment of unstable vitiligo. Dermatology 2015; 231(3): 286.

23. Singh A, Kanwar AJ, Parsad D, Mahajan R. Randomized controlled study to evaluate the effectiveness of dexamethasone oral minipulse therapy versus oral minocycline in patients with active vitiligo vulgaris. Indian J Dermatol Venereol Leprol 2014; 8029.

24. Siadat AH, Zeinali N, Iraji F, Naeini BA, Nilforoushzadeh $M A$, Jamshidi $K$ et al. Narrow-band ultraviolet $B$ versus oral minocycline in treatment of unstable vitiligo: a prospective comparative trial. Dermatol Res Pract 2014; 1.

25. Shi Q, li K, Fu J, Wang Y, Ma C, Li Q et al. Comparison of 308-nm excimer laser with the 308-nm excimer lamp in the treatment of vitiligo-a randomized bilateral comparison study. Photodermatol Photoimmunol Photomed 2013; 29: 27.

26. Lim HW, Grimes PE, Agbai O, Hamzavi I, Henderson M, Haddican $\mathrm{M}$ et al. Afamelanotide and narrowband UV-B phototherapy for the treatment of vitiligo: a randomized multicenter trial. JAMA Dermatol 2015; 151: 42.

27. Nisticò S, Chiricozzi A, Saraceno R, Schipani C, Chimenti S. Vitiligo treatment with monochromatic excimer light and tacrolimus: results of an open randomized controlled study. Photomed Laser Surg 2012; 30: 26. 
28. Ṭabrī A, Ḥasan A bin M. Al-Mu, ālajat al-Buqrāțiya (VolII). (Central Council For Research in Unani Medicine, New Delhi) 1997, 199.

29. Ibn Sīnā al-S al-RA, Ali al-H ibn, Abd A, Al-Qānūn fi"I Tibb. (1st ed. Kantoori GH, editor. Shaikh Muhammad Basheer and Sons, Urdu Bazar, Lahore) 351.

30. Jurjāni $A H$, Dhakhīra Khawārizm Shāhī, Khan $\mathrm{HHH}$, editor. (Idara Kitab-us-Shifa, 2075, Kucha Chelan, Darya Ganj, New Delhi) 8(18) 2010, 10.

31. Arzānī HA, Ṭibb-i Akbar, Husain AHM, editor. 247554 (Faisal publications, Jama Masjid, Deoband), 731.

32. Gāzrūnī S al-D, Al-Sadīdī (Arabic), (Mațba Munshi Nawal Kishor, Lucknow) 61.

33. Standard Unani Medical Terminology, (Central Council for Research in Unani Medicine, New Delhi) 2012, 32.

34. Rāzī ABM ibn Z, Kitāb al-Mansūri (Urdu translation), (Central Council for Research in Unani Medicine, New Delhi) 1991, 207.

35. Hakeem AH, Bustan al-mufradat, Idara Kitab-us-Shifa; New Delhi) 2002, 109. 171, 249, 340, 417, 419, 431.

36. Khan MA, Muheete azam, vol. III. (CCRUM, New Delhi) 2014, 107, 419.

37. Ghani N, Khazain al-adviya, Idara Kitab-us-Shifa (New Delhi) YNM, 174, 316, 474, 635, 815, 1007.

38. Dilshad Ali, Mannan A, Misbahuddin S. Bars aur uskey ilaj mein safoof bars ki ifadiat ka jaiza. Unani Medicus - An International Journal. (Aligarh Muslim University) 2015, 2.

39. Arzani MA. Keemiae anasri (Urdu translation of Qarabadeene Qadri by Noor Kareem HM), (CCRUM, New Delhi) 2006, 752, 747.

40. Barton BL. International Conference on HarmonizationGood Clinical Practices Update. Drug Information Journal 1998; 32(4) 1143.

41. Bradley DW, Maynard JE, Emery G, Webster H. Transaminases activities in serum of long term hemodialysis patients. Clin Chem 1972; 18(11): 1442.

42. Tiffany TO, Jansen JM, Burtis CA. Overton JB and Scott $C D$, Enzymatic kinetic rate and end-point analyses of substrate, by use of a GeMSAEC fast analyzer. Clin Chem 1972; 18(8): 829.

43. Bowers LD. Kinetic serum creatinine assays I, The role of various factors in determining specificity. Clin Chem 1980; 26(5): 551.

44. Pearlman, Lee RT. Detection and measurement of total Bilirubin in Serum, with Use of surfactants as solubilizing Agents. Clinical Chemistry 1974; 20: 447.

45. Trinder PJ. Clin Pathol 1949; 22: 246.

46. Mukherjee KL. Medical Laboratory Technology, 3rd Edition, (Tata Mc Graw-Hill Publishing Company Limited, New Delhi), 1990, 228.

47. Shah H, Mehta A, Astik B. Clinical and sociodemographic study of vitiligo. Indian J Dermatol Venereol Leprol,
2008; 74(6): 701.

48. Virendra NS, Govind S. Vitiligo: Compendium of clinicoepidemiological features. Indian J Dermatol Venerol Leprol 2007; 73: 149.

49. Almomani N, Abdulmajeed I, Ur Rahman S, Alakkam A. Quality of Life and Affective Health of Patients with Vitiligo. J Sleep Disord Ther 2015; 4(2): 1.

50. Yoon J, Sun YW, Kim TH. Complementary and Alternative Medicine for Vitiligo, Vitiligo-Management and Therapy, Dr. Kelly Kyung Hwa Park (Ed.), ISBN: 978-953-307731-4, InTech, (InTech China Unit 405, Office Block, Hotel Equatorial Shanghai No.65, Yan An Road (West), Shanghai, 200040, China) (2011) PP-143.

51. Thulasamma S, Chakraborty A, Jamil K, Kazmi MH, Kareemullah S, Ahmad A. Molecular and genetic basis of vitiligo-Concept of melanogenesis in Unani medicine. WJPPS 2017; 6(12): 1241.

52. Mahajan VK, Vashist S, Chauhan PS, Mehta KIS, Sharma V, Sharma A. Clinico-Epidemiological Profile of Patients with Vitiligo: A Retrospective Study from a Tertiary Care Center of North India. Indian Dermatol Online J 2019; 10(1): 38.

53. Khaitan BK, Kathuria S, Ramam M. A descriptive study to characterize segmental vitiligo. IJDVL 2012I; 78(6): 715.

54. Verma RS, Khan P, Mohammad N, Khan LA. A clinical study of Unani formulation UNIM-045 for Anti-Vitiligo (Bars) effect. Hippocratic Journal of Unani Medicine 2012; 7: 31.

55. Rehman S, Verma RS, Khan SA, Khan LA. The efficacy and safety of Unani coded drug UNIM-046 in cases of Bars (Vitiligo)- A preliminary clinical trial. Hippocratic Journal of Unani Medicine 2012; 7: 11.

56. Verma RS, Abbas A, Abbas S, Khan LA. Efficacy of Unani Coded drug UNIM-044 in Vitiligo (Bars) patients-A clinical study. Hippocratic Journal of Unani Medicine 2011; 6: 23.

57. Husain N, Uddin Q, Kazmi MH. Clinical studies on the treatment of Bars (Vitiligo)in Unani system of mrdicine-A systematic review. EJBPS 2018; 5(5): 1088. 